\title{
Environmental Policy and Firm Investment Behaviour when Energy Saving Technologies are Available
}

\author{
Emanuela Giusi Gaeta* \\ University of Rome "Tor Vergata”, Rome, Italy \\ E-mail: gaeta@juris.uniroma2.it \\ Received June 8, 2010; Revised July 6, 2010; accepted July 12, 2010
}

\begin{abstract}
This paper investigates upon the effects of taxation on firm investment behaviour in presence of alternative energy (oil) saving technologies and scarce resources in competitive markets. Socially optimal policies are compared to a decentralized regulatory framework: the paper shows that taxation affects the adoption of different energy saving technologies hence the aggregate amount of energy saving. To our knowledge, there are few works that underline the relationship between environmental policies and firms' incentives to adopt oil saving technologies. For this reason, we follow the theoretical literature focusing on the effects of environmental policy applied to pollution and climate change by adapting it to the energy saving perspective. We perform a static comparison of environmental policies to show that different levels of the same instrument lead to different results in terms of the number of firms adopting energy saving technologies; multiple equilibrium are possible but there is only one which is socially optimal.
\end{abstract}

Keywords: Environmental Policies, Fiscal Policies, Technological Change, Energy Saving, Welfare Analysis

\section{Introduction}

In the last decade, debates around environmental economics and policy have become increasingly permeated by issues related to technological change. One aspect of this issue relates to the effects that environmental policies have on firms' behaviour. In particular the literature has focussed on the comparison of alternative policy instruments targeted to reduce social costs, paying specially attention to pollution and climate change, rather than to energy saving. There are few works underlining the relationship between environmental policy and firms' incentives to adopt oil saving technologies and this paper follows such research line. In general, a large number of papers aim at establishing a ranking between alternative environmental policy instruments regarding the extent to which they enhance the adoption and diffusion of pollution control technology both at industry level [1-3] and firm level [4]. The goal in evaluating the different instruments is to compare the cost before and after adoption of the abatement technology, where the latter differs from a conventional "dirty" technology in terms of a lower marginal abatement cost [5]. However the literature is rather

\footnotetext{
*I am indebted to the IAD - University of Rome Tor Vergata (http: www.
} scuolaiad.it) that provides funds for my research. scattered: some authors focus on the ranking of market-based instruments by taking into account the time inconsistency of the environmental policy related to the delay between announcement and actual implementation $[6,7]$. Other authors investigate upon the effect that uncertainty - on the arrival time of the advanced technology - plays on firms' choice, distinguishing by the case where the regulator anticipates or not the arrival of new technology.

Unlike the cited literature, this paper investigates upon the effects of taxation on firm investment behaviour in presence of alternative energy (oil) saving technologies and scarce resources in competitive markets. Socially optimal policies are compared to a decentralized regulatory framework: the paper shows that taxation affects the adoption of different energy saving technologies hence the aggregate amount of energy saving and decentralized policies are succesfull in achieving first best outcome $[8,9]$. The paper is strongly inspired by the theoretical analysis of Requate and Unold [4], but their research is focused on the comparison of policy instruments (taxes, auctions permits and standards) to reduce pollution in competitive markets rather than investigate upon the adoption of oil-saving technologies. They show that different policy instruments lead to quite different results in terms of emissions, if the number of firms which adopt 
the new technology is determined endogenously through equilibrium consideration.

Following their research, we try to define the optimal policy by comparing "market-based instruments", to a "command-and-control" policy. Unlike the recent literature, we do not assume that all firms, belonging to the same industry, will adopt the new technology. Following Requate and Unold [4], we endogenize the number of adopting firms by analysing two scenarios that differ with respect to the order of first mover in a two-stage game: regulator-by setting the level of his policy instruments (e.g. tax rate) — or the firm by investing.

In the first one, called ex-ante optimal policy, we assume that the regulator makes a commitment both to the choice and level of his policy instrument. In this case the regulator moves at first, while the firms invest in the second stage, after observing the new tax rate and decide for the possible adoption of the new technology. In the second strategy, named ex post optimal policy, we are going to assume that, before the game starts, the regulator makes a commitment to the choice of his policy instrument only, although we are focusing on taxation only. Then she observes how many firms invest in the first stage and finally she chooses the level of the instrument. In the first case, for each implementation $\cos t F$, a particular number of firms should invest for an optimal outcome. This would require coordination among firms. Since firms, however, are indifferent between investing and not when the tax rate equals the socially optimal marginal damage - which is a necessary condition for optimality-inefficient outcomes are likely to occur in this case. So, for the first scenario, we confirm the result found by Requate, Unold [4]: there are multiple equilibria, a lot of these are inefficient and the runic solution that can be pursued is the second best.

In the second situation, the planner moves after observing firms choice. In such a way the first best policy is a sub-game perfect equilibrium. Nevertheless, the implementation of such an equilibrium depends on the way the game is played between firms and planner. In such a case, Requate and Unold [4] find that there exists a unique subgame perfect equilibrium where the optimal number of firms invests and the regulator chooses the optimal tax rate. But firms move simultaneously and in an uncoordinated way, so nothing assures that the optimal number of firms investing in the advanced technology (first best) will be, in fact, implemented. Unlike Requate and Unold, [4], we are going to show that, if players act in a different way, the solution of first best will be implemented in a decentralized economy. This is possible in a "multi-stage game with observed actions and perfect information" [10] where the planner moves at the last stage and backward induction is a viable solution. This result can be considered as the original aspect of our paper (a previous inves- tigation is found in Gaeta [11]), which makes a little but substantial difference with the research made by Requate and Unold [4].

This paper is organized as follows: In the next section, we discuss the characteristics of the model. Section 3 describes firms' behaviour. In Section 4 we present the social optimum and derive the socially optimal allocation. In Section 5 we study two scenarios, in the first the regulator announces the instrument and fixes the relative level, while in the second she announces the instrument, but fixes the instrument in a second stage, after observing the firm's behaviour. In Section 6 we present a numerical example which summarises the model. Conclusions follow in Section 7. Technical proofs are found in Appendix.

\section{The Model}

There are $n$ small firms operating in a competitive market. Every firm produces by means of a production function characterized by DRS, where the argument is oil $(O)$. Under a laisser-faire policy, the firms choose the first best quantity $O_{\max }$. Taxation on oil makes it expensive and firm are induced to substitute oil with an alternative input: $e$.

The firms can choose between two technologies: the first one, that we call conventional technology, is oil intensive, while the second one, called advanced technology, is "oil saving". This means that, for the same output level, the advanced technology uses a lower level for $O$. However, the implementation of the second technology is costly, according to a sunk cost $F$. We are going to assume that each firm starts with the conventional technology.

We assume that oil price is given. This is so because the domestic oil demand is not able to affect the international oil price.

$O_{\max }$ is the oil usage in the first best equilibrium, i.e. when the firms operate without taxation. With oil taxation, the level $O$ chosen by the firm can be reduced throughout an alternative input $e$. The $e$ usage depends on $O, e(O)$, with $e^{\prime}(O)<0, e \in\left[0, e_{\max }\right]$. $C_{e}(e)$ is the usage cost for $e$ with $C_{e}\left(e_{\max }\right)=\infty$, $C_{e}(0)=0$. It is helpful using $O$ as the unit of reference for the model and expressing $C_{e}(e)$ in terms of $O$; so we call $C(O)$ the substitution cost, between $e$ and $O$, in terms of $O$. Given the relationship $e(O)$, we conclude that $C\left(O_{\max }\right)=0,-C^{\prime}(O)>0, C(0)=\infty$

Figure 1 highlights the relationship between $C_{e}(e)$ and $C(O)$. 
The picture must be read clockwise: starting from a given $O \in\left[0, O_{\max }\right]$ in the first diagram (e.g. $\mathrm{C}$ in the picture), we can obtain the implied level of $e$ in the second one (the $\mathrm{D}$ point); the latter shows the assumed linear inverse relationship between $e$ and $O$. The 45 degree line in the third diagram brings the point $\mathrm{D}$ in the fourth diagram. The latter shows the $C_{e}(e)$ cost function. By linking the $O$ quantity with $C_{e}(e)$ we obtain a point in $C(O)$; by iterating the proces we obtain the cost function in the $O$ space depicted in the first diagram.

Without taxation, the firm chooses the first best level of $O=O_{\operatorname{Max}}$ by using the conventional technology; this involves $e=0$ (point A).

When two technologies are available the situation is the one shown in figure two. The first technology, that we call "conventional", labeled 0 , is immediately available to firms free of additional implementation costs. The second one, that we call "advanced" and labelled 1, is more efficient and allows firms to save $e$ for each level of $O$ with respect the conventional technology; however firms adopting such a technology incur in a sunk cost $F$. Figure 2 shows that $O_{M a x}^{1}<O_{M a x}^{0}$ and that $\forall O<O_{\text {Max }}^{1} \rightarrow C^{1}(O)<C^{0}(O)$. As an example, for a given $O$ oil usage we have $C^{0}(O)>C^{1}(O)$.

Given the assumptions on the cost functions, the marginal oil saving costs $M C=C^{\prime}(O)$ have the following characteristics: $-C_{i}^{\prime}(O)>0 \forall O<O_{\text {Max }}^{i}, \quad i \in\{0,1\}$ and strictly increasing in oil saving, $C_{i}^{\prime \prime}(O)>0 \quad \forall O<O_{\text {Max }}^{i}$, $i \in\{0,1\}$. In other words, everytime the firm reduces the quantity $O$, the $\mathrm{MC}$ increases. It is worth stressing that, for the same $M C$, i.e. $M C_{O}=M C_{1}$, we have $O_{1}<O_{0}$.

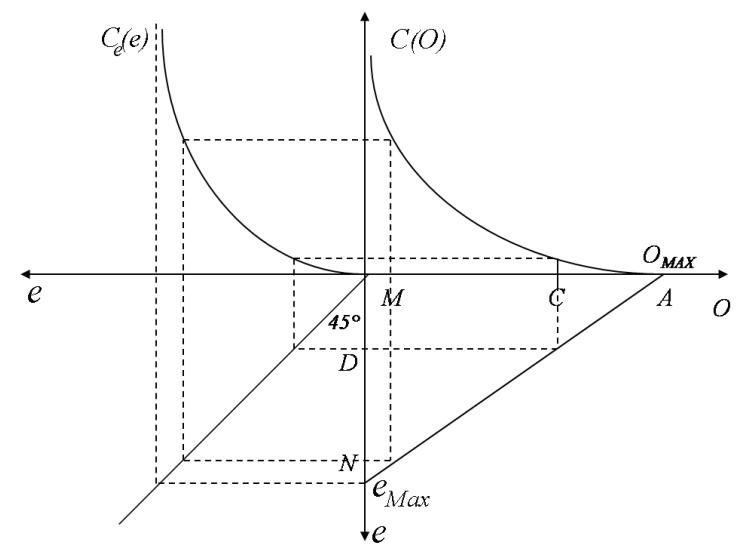

Figure 1. Energy saving costs with one technology.

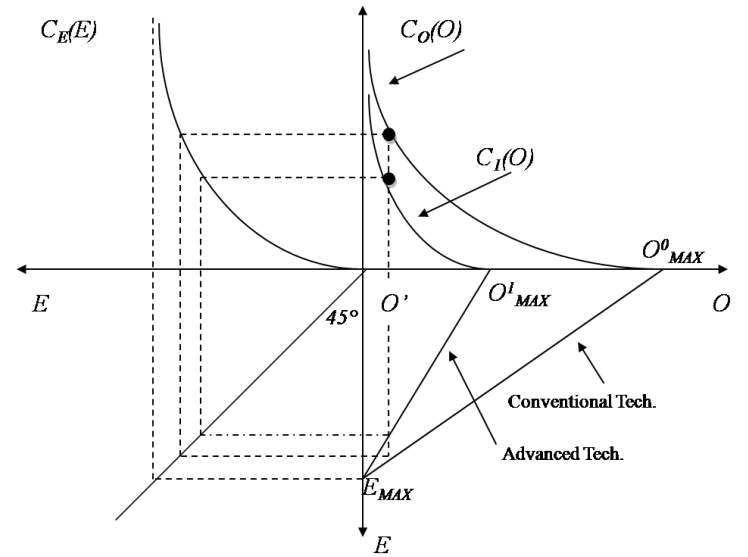

Figure 2. Energy saving costs with two technologies.

\section{Equilibrium in Competitive Markets}

We know that the solution of first best under competitive market conditions is to produce with the quantity $O_{\operatorname{Max}}$.

The profit function is:

$$
V(O)=P Q\left(O_{\max }\right)-P_{o} O_{\max }
$$

where $P$ is the output price, normalized to one and $Q\left(O_{\max }\right)$ is the quantity in the first best situation. $P_{o} O_{\max }$ is the cost of using $O_{\max }$ at the international price $P_{o}$.

When oil taxation is at work, profits are as follows:

$$
V(O)=P Q(O, e(O))-P_{o} O-C(e(O))-\tau O
$$

where $Q(O, e(O))$ is the output quantity which depends on $O$ troughout $e$, while $C(e(O))$ is the cost for oil saving and $\tau O$ is the fiscal burden.

Let us rewrite the previous equation for the two technologies in this way:

$$
\begin{gathered}
V\left(O_{0}\right)=\pi\left(O_{0}\right)-C\left(e\left(O_{0}\right)\right)-\tau O_{0} \\
V\left(O_{1}\right)=\pi\left(O_{1}\right)-C\left(e\left(O_{1}\right)\right)-\tau O_{1}-F
\end{gathered}
$$

where $F$ is the sunk cost of adopting the advanced technology. $\pi\left(O_{i}\right)$ is the profit gross of tax and adjumest cost. By deriving with respect to $O_{i}, i \in\{0,1\}$ we obtain the following F.O.C.:

$$
\begin{aligned}
& \frac{\partial \pi_{0}\left(O_{0}\right)}{\partial O_{0}}-\frac{\partial C_{0}\left(O_{0}\right)}{\partial O_{0}}=\tau \\
& \frac{\partial \pi_{1}\left(O_{1}\right)}{\partial O_{1}}-\frac{\partial C_{1}\left(O_{1}\right)}{\partial O_{1}}=\tau
\end{aligned}
$$

By taxing oil usage, the government induces firms to adopt or not the advanced technology. Now we are going to show that does exist only one tax rate that leaves firms indifferent in adopting or not. 
By assuming technology satisfies $\pi\left(O_{0}\right)=\pi\left(O_{1}\right)$, for a fixed $\tau$, firms are indifferent to the adoption of new technology if:

$$
C_{0}\left(O_{0}(\tau)\right)+\tau O_{0}=C_{1}\left(O_{1}(\tau)+\tau O_{1}+F\right.
$$

where $O_{i}, i \in\{0,1\}$ is the optimal oil usage under technology 0 and 1 respectively. The indifference condition is quite simple: it claims that firms are indifferent when the costs of oil saving, inclusive of taxation, are equal under both technologies.

Lemma 1 shows that does exist $\tau$ satisfating equation indifference:

Lemma There exists at most one tax rate, $\widetilde{\tau}$, such that firms are indifferent between adopting and not adopting the new technology. For this $\tilde{\tau}$, there exists a unique oil quantity $\widetilde{O}_{1}$ and $\widetilde{O}_{0}$ chosen by the firms with and without advanced technology, respectively. If $\tau>\tilde{\tau}(\tau<\tilde{\tau})$ all firms (no firm) want to adopt the new technology. Moreover, $\tilde{\tau}$ is increasing in $F$.

The proof is given in Appendix. Rationale is straightforwad. If $\tau<\widetilde{\tau}$, the taxation is too low to induce firms to afford the sunk cost $F$ in order to adopt the advanced technology; no firm chooses to do that. The result is the maximum oil wasting in the economy, as all firms remain on the inefficient technology: it amounts to $\bar{O}=n \widetilde{O}_{0}$, where $n$ is the number of firms in the economy. Conversely, when taxation is sufficiently high, $\tau>\widetilde{\tau}$, it is too costly remaining with the conventional technology and all firms are induced to adopt the advanced one. In such a case the oil usage in the economy is lower than the previous case $O=n \widetilde{O}_{1}$. Lemma 1 shows that between these two opposite case there exists an equilibrium of partial adoption, where firms are indifferent to adopt or not $\tau=\tilde{\tau}$. The number of adopting firms is undetermined and the total oil usage in the economy lies in the closed set $O \in[\underline{O}, \bar{O}]$.

\section{Social Optimum}

The previous lemma shows that the government is able to affect firms choice about the adoption of the technology. The amount of oil wasting in the economy is under the indirect control of central authority. If the government goal were to reduce the oil wasting in the economy it should fix a higher tax rate; as lemma 1 shows, in such a case, all firms invest in the new technology. Nevertheless this involves a private cost $F$ for each firm that reduces the social welfare. There is a trade-off between the private cost of increasing tax and the social benefit of oil saving induced by a high taxation. Hence, the social planner's problem is to minimize the social costs by balancing in- dustry's total saving costs against the damage caused by the oil wasting. To do so, it chooses the optimal taxation filling the goal; this involves an optimal aggregate oil usage in the economy and a corresponding optimal number of adopting firms $n_{1}^{*}$. The social equilibrium is given by the triple $\left\{O_{o}^{*}, O_{1}^{*}, n_{1}^{*}\right\}$.

The social cost can be represented by a loss function $D(W)$, with $W=n_{1} O_{1}+\left(n-n_{1}\right) O_{0}$, which evaluates the aggregate oil wasting in monetary terms. This implies that only aggregate oil wasting matters [4]. We assume that the damage function is increasing and convex in $W$, i.e. $D^{\prime}(W)>0$ and $D^{\prime \prime}(W)>0$. If the regulator wants to minimize the social cost, she has to resolve this equation:

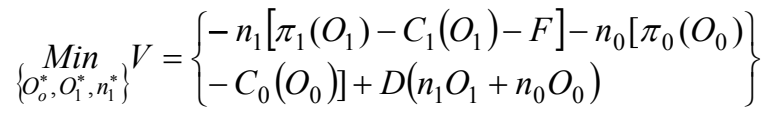

The socially optimal allocation can be characterized as follows:

Proposition There is an interval of fixed costs $[\underline{F}, \bar{F}]$ such that:

1) No firm should adopt the new technology for $F>\bar{F}$;

2) All the firms should adopt the new technology for $F<\underline{F}$;

3) For $F \in[\underline{F}, \bar{F}]$ partial adoption is optimal.

The optimal number of firms $n_{1}^{*}$ adopting the new technology is decreasing in $F$. The optimal marginal damage $M D^{*}(F)$ is decreasing in $F$.

The proof is given in Appendix. The intuition is that if the fixed cost $F$ of installing the advanced technology is extremely high, firms do not adopt this technique. Conversely, if $F$ is sufficiently low, all the firms should adopt the new technology. In both cases, the optimal aggregate oil wasting $\underline{O}$ and $\bar{O}$ are independent of $F$.

\section{Optimal Policy: Two Possible Scenarios}

In this last section we are going to analyze how social planner can implement the socially optimal equilibrium. We are doing so by following two possible strategies. In the first one, called ex-ante optimal policy, we assume that the regulator makes a commitment both to the choice and level of his policy instrument. In this case the regulator moves at first, while firms invest in the second stage, after observing the tax rate, and decide for the possible adoption of the new technology. This is summarized in the following proposition: 
Proposition (Ex ante optimal policy). If the welfaremaximizing regulator moves first, then: for $F<\underline{F}$ and $F>\bar{F}$, there exits a unique subgame perfect equilibrium leading to a first best outcome. For $F \in[\underline{F}, \bar{F}]$ there are multiple equilibria. One of these equilibria is efficient but there are also many inefficient equilibria with too little and too much investment.

(See Appendix)

In the second strategy, ex post optimal policy, we are going to assume that, before the game starts, the regulator makes a commitment to the choice of his policy instrument only, so she observes firms investing in the first stage, then she chooses the level of the instrument in the second stage. Unlike the previous case, with such a strategy the socially optimal equilibrium can be implemented in the economy.

Proposition (ex post optimal policy). If the regulator sets his optimal policy after observing the number of investing firms, then under taxes there is a unique subgame perfect equilibrium where the optimal number of firms invests, and the regulator sets the first best tax rate.

The first part of the proposition follows Requate and Unold [4]. In a two stage game with informed and rational players, we can use the backward induction for proving the statement. In fact, firms know that, in the second stage of the game, the governement is going to set the optimal tax rate coherently with the optimal number of adopting firms $n_{1}^{*}$ (first best). If $n_{1}^{*}$ is chosen by firms in the first stage of the game, then this is a sub game perfect equilibrium as neither firms nor planner has an incentive to modify their choice. If firms choose $n_{1}>n_{1}^{*}$, then the best strategy of the planner is to set the tax rate conditional on $n_{1}$, i.e. $\tau\left(n_{1}\right)<\tau^{*}\left(n_{1}^{*}\right)$ in the second stage, as the social damage reduce with respect to $n_{1}$ and tax must be reduced consequently. But this means that some firms that was adopting in stage 1 would be better off from not adopting, as the tax rate is lower than expected. So we conclude that $n_{1}$ is not an equilibrium. Same result holds when $n_{1}<n_{1}^{*}$. Nevertheless, the game analyzed by Requate and Unold [4] shows only that an equilibrium exists under subgame perfection, but this does not imply that such equilibrium will be effectively implemented. Unlike Requate and Unold [4], we are going to show that, if players act in a different way, $n_{1}^{*}$ will be implemented in a decentralized economy. This is possible in a "multi-stage game with observed actions and perfect information" [10]. In such a game, the choice set of each player is enlarged by the move "do nothing". As an example, any single firm strategy set, per each game stage, is composed by adopting, not adopting, "do nothing"\}. Moreover, any player knows the history of the game, as everyone observes past actions.

We say that a multi-stage game has perfect information if, for every stage $k$ and history $h^{k}$, exactly one player has a nontrivial choice set-a choice set with more than one element - and all the others have the one-element choice set "do nothing" [10].

The planner moves at the last stage, so backward induction is still a viabile solution. Everyone knows the optimal taxation level chosen by the planner at the last stage of the game as consequence of $n_{1}^{*}$. The game is composed by $n+1$ stages. At each stage one firm chooses whether adopting or not, knowing what happened up to now; the other one choose "do nothing". Once the single firm has played either "adoption" or "not adoption" it will choose "do nothing" in the rest of the game. In such a way, when $n_{1}^{*}$ has been achieved in the economy it becomes common knowledge, as everyone knows the past history of the game. From now and on, rational firms do choose "not adopting" as they know that if $n_{1}>n_{1}^{*}$ the taxation rate chosen by the planner at the last stage is not choerent with their choice, following the reasoning of the sub-game perfection used for the first part of the proposition..

\section{A Numerical Example}

In this section we discuss a numerical example to show in detail the mechanism of the model.

We know that the firm can choose between two techniques that are characterized by different costs.

For each technology, the firm supports an energy saving cost: $C_{0}(O), C_{1}(O)$. We suppose that this kind of cost functions has the following explicit form, choerently with the assumptions described in Section 1:

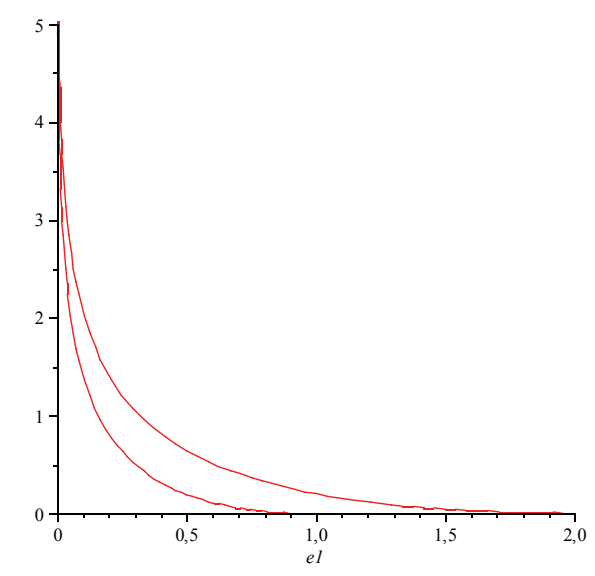

Figure 3. Oil saving costs. 


$$
\begin{gathered}
C_{0}(O)=0.5\left(O_{0}\right)-\ln \left(O_{0}\right)-0.3 \text { "oil intensive" } \\
C_{1}(O)=O_{1}-\ln \left(O_{1}\right)-1 \text { "advanced technology" }
\end{gathered}
$$

These functions are depicted in Figure 3, which corresponds to the graph of the first diagram reported in Figure 2.

As shown by the graph, for each technique, we obtain a monotonic decreasing function on its closed domain, that describes the negative relationship between the energy saving costs: $C_{o}(O), C_{1}(O)$ and the oil quantity.

The marginal energy saving costs: $C_{o}^{\prime}(O)$ and $C_{1}^{\prime}(O)$ are described by the two functions presented in Figure 4, where, $C_{1}^{\prime}(O)=1-\frac{1}{O}$ and $C_{o}^{\prime}(O)=0,5-\frac{1}{O}$ and they respect the assumptions made in Section 2.

Now, we give a numerical explanation of Lemma 1.

The Figure 5 shows that for a fixed $\mathrm{F}$, there exists at most one tax rate, such that firms are indifferent between adopting and not adopting the new technology. In fact, for $F=1.4142$ there is only intersection point that individuates: $\bar{\tau}=0,66$ that satisfies the indifference condition.

If we vary $F$, we can observe the positive relation between $F$ and $\tilde{\tau}$, as shown by Table 1 .

By proposition 2, we know that if the new technology is available, the social planner seeks to minimize total social costs expressed by the equation lagra which is a numerical example of the equation lagrth.

By assuming $Q\left(O_{i}\right)=2\left(O_{i}\right)^{0.7}+2$ the Lagrangian that solves this problem is reported in the following equation:

$$
\begin{aligned}
& L=-n_{1}\left[\left(3-3 O_{1}+2\left(O_{1}\right)^{0.7}+\ln \left(O_{1}\right)-F\right]-\left(1-n_{1}\right)\right. \\
& {\left[1.3-2.5\left(O_{0}\right)+2\left(O_{0}\right)^{0.7}+\ln \left(O_{0}\right)\right]+\left[n_{1} e_{1}+\left(1-n_{1}\right) e_{0}\right]^{2}}
\end{aligned}
$$

This numerical example shows the existence of the values of first best: $O_{o}^{*}, O_{1}^{*}, n_{1}^{*}$.

The Table 2 shows the content of proposition 2 .

If we vary $F$, we can observe that there is an interval of fixed costs $[\underline{F}, \bar{F}]$ such that for:

$1,445<F<1,458$ we obtain $n_{0}, n_{1}>0$, so there is partial adoption, while for $F \leq 1,445$ or $F \geq 1,458$ we observe corner solutions.

\section{Conclusions}

The paper shows the existence of an optimal policy that reduces the social cost represented by oil usage. The regulator can implement this policy throughout two alternative strategies: the ex ante optimal policy or the ex post optimal policy. The choice depends on the sunk cost of

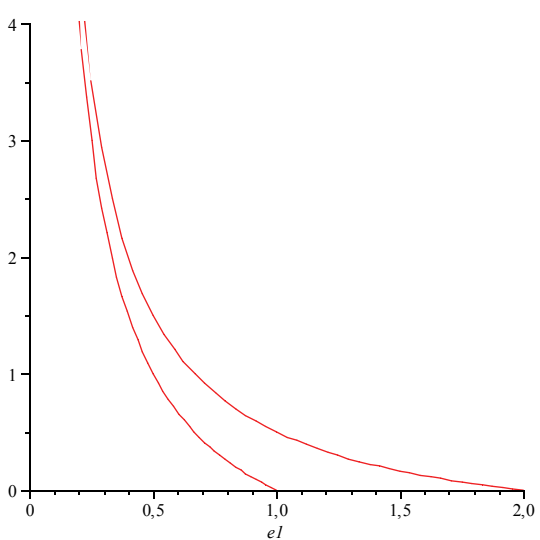

Figure 4. Marginal energy saving costs.

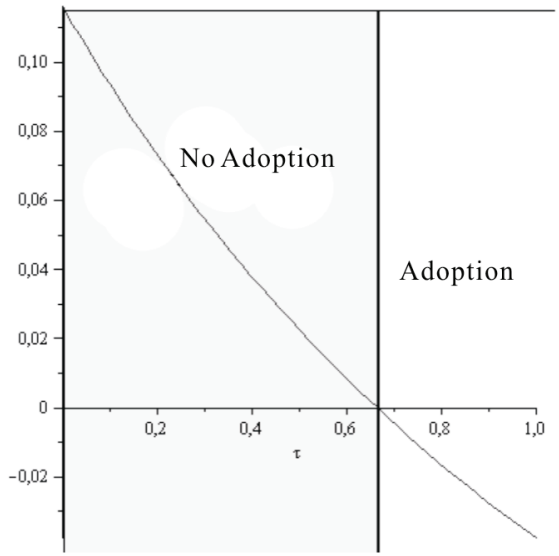

Figure 5. Lemma 1.

Table 1. Relationship between $F$ and $\tilde{\tau}$.

\begin{tabular}{cllllll}
\hline$F$ & 1,3 & 1,33 & 1,36 & 1,39 & 1,42 & 1,45 \\
\hline$\widetilde{\tau}$ & 0,008 & 0,14 & 0,30 & 0,49 & 0,71 & 0,98 \\
\hline
\end{tabular}

Table 2. Proposition 2.

\begin{tabular}{lcccc}
\hline$O_{0}$ & $O_{1}$ & $F$ & $n_{1}$ & $\tilde{\tau}$ \\
\hline 0,000 & 0,463 & 1,445 & 1 & 0.832 \\
0,563 & 0,460 & 1,446 & 0,9 & 0.842 \\
0,559 & 0,458 & 1,447 & 0,8 & 0.851 \\
0,559 & 0,458 & 1,448 & 0,8 & 0,861 \\
0,555 & 0,455 & 1,449 & 0,7 & 0,871 \\
0,552 & 0,453 & 1,450 & 0,6 & 0,881 \\
0,552 & 0,453 & 1,451 & 0,6 & 0,891 \\
0,549 & 0,450 & 1,452 & 0,5 & 0,900 \\
0,545 & 0,448 & 1,453 & 0,4 & 0,912 \\
0,542 & 0,446 & 1,454 & 0,3 & 0,922 \\
0,542 & 0,446 & 1,455 & 0,3 & 0,932 \\
0,539 & 0,444 & 1,456 & 0,2 & 0,943 \\
0,536 & 0,442 & 1,457 & 0,1 & 0,953 \\
0,533 & 0,000 & 1,458 & 0 & 0,964 \\
\hline
\end{tabular}


adopting the oil saving technology. If the implementation cost is outside the range $[\underline{F}, \bar{F}]$ the regulator is indifferent between the two strategies: both strategies lead to the first best. If the implementation cost is within the range, the regulator can implement the optimal policy only by choosing the ex post optimal policy, where firms move according to a multi-stage game with informed and rational players. So the paper confirms that policies ruled by the market are viable instruments for environmental control. We also show that, unlike the current literature, first best solution is actually implemented when firms and the regulator play a sequential game.

The sunk cost $F$ plays a leading role for results; in a following paper we are going to investigate how results change when such a cost is made endogenous by assuming an $\mathrm{R} \& \mathrm{D}$ sector in the economy.

\section{References}

[1] P. B. Downing and L. J. White, "Innovation in Pollution Control," Journal of Environmental Economics and Management, Vol. 13, No. 1, 1986, pp. 18-29.

[2] S. R. Milliman and R. Prince, "Firms Incentives to Promote Technological Change in Pollution Control," Journal of Environmental Economics and Management, Vol. 17, No. 3, 1989, pp. 247-265.

[3] Ch. Jung, K. Krutilla and R. Boyd, "Incentives for Advanced Pollution Abatement Technology at the Industry Level: An Evaluation of Policy Alternatives," Journal of
Environmental Economics and Management, Vol. 30, No. 1, 1996, pp. 95-111.

[4] T. Requate and W. Unold, "Environmental Policy Incentives to Adopt Advanced Abatement Technology: Will the True Ranking Please Stand up?" European Economic Review, Vol. 47, No. 1, 2003, pp. 123-146.

[5] T. Requate, "Dynamics and Incentives by Environmental Policy Instruments-A Survey," Ecological Economics, Vol. 54, No. 2-3, 2005, pp. 175-195.

[6] D. P. van Soest, "The Impact of Environmental Policy Instruments on the Timing of Adoption of Energy-Saving Technologies," Resource and Energy Economics, Vol. 27, No. 3, 2005, pp. 235-247.

[7] A. von Döllen and T. Requate, "Environmental Policy and Incentives to Invest in Advanced Abatement Technology if Arrival of Future Technology is Uncertain," Economics Working Paper No 2007-04, 2007.

[8] J. Montero, "Permits, Standards, and Technology Innovation," Journal of Environmental Economics and Management, Vol. 44, No. 1, 2002, pp. 23-44.

[9] C. Carraro and D. Siniscalco, "Environmental Policy Reconsidered: The Role of Technological Innovation," European Economic Review, Vol. 38, No. 3-4, 1994, pp. 545-554.

[10] D. Fudenberg and J. Tirole, "Game Theory," MIT Press, Cambridge, 1991.

[11] E. G. Gaeta, "Environmental Policy, Innovation and Adoption of Energy Saving Technologies," Phd Dissertation, University of Rome, "La Sapienza", 2009. 


\section{Appendix}

\section{Proof of Lemma 1}

Now we focus on the total difference of the net value of the i- th firm:

$$
\Delta V(O)=V_{0}\left(O_{0}\right)-V_{1}\left(O_{1}\right)
$$

So we can write A.1 as follows:

$$
\begin{aligned}
& \Delta V(O)=\left[\pi_{0}\left(O_{0}(\tau)\right)-C_{0}\left(O_{0}(\tau)\right)-\tau O_{0}(\tau)\right] \\
& -\left[\pi_{1}\left(O_{1}(\tau)\right)-C_{1}\left(O_{1}(\tau)\right)-\tau O_{1}(\tau)-F\right]
\end{aligned}
$$

We differentiate A.2 respect to $\tau$ to demonstrate that there exists at most one tax rate such that firms are indiferent to adption or not adoption.

$$
\begin{aligned}
& \frac{\partial \Delta V(O)}{\partial \tau}=\left[\frac{\partial \pi_{0}}{\partial O_{0}} \frac{\partial O_{0}}{\partial \tau}-\frac{\partial C_{0}}{\partial O_{0}} \frac{\partial O_{0}}{\partial \tau}\right]-O_{0}(\tau)-\tau \frac{\partial O_{0}}{\partial \tau} \\
& -\left\{\left[\frac{\partial \pi_{1}}{\partial O_{1}} \frac{\partial O_{1}}{\partial \tau}-\frac{\partial C_{1}}{\partial O_{1}} \frac{\partial O_{1}}{\partial \tau}\right]-O_{1}(\tau)-\tau \frac{\partial O_{1}}{\partial \tau}\right\}
\end{aligned}
$$

substituting Equations (1), (2) into A.3 we obtain:

$$
\frac{\partial \Delta V(O)}{\partial \tau}=O_{1}(\tau)-O_{0}(\tau)<0
$$

which is always negative, because as previously stressed, the same $\mathrm{MC}$ involves $O_{1}<O_{0}$.

When $\tau=0 \rightarrow \Delta V(O)=F>0$, since $\pi_{0}\left(O_{0}\right)=\pi_{1}\left(O_{1}\right)$ by the competitive market assumtion. We know that $\Delta V(O)$ is a monotonic function and it decreases if $\tau$ increases, as A.4 shows; this means that there is a unique $\tau$ called " $\bar{\tau}$ " which ensures that firms are indifferent between the two technologies, i.e. when $\Delta V(O)=0$.

Now we demonstrate that $\tilde{\tau}$ is increasing in $F$.

Differentiating the net value A.2 with respect to $F$ and remembering the F.O.C., we obtain:

$$
\begin{gathered}
\frac{\partial \tau}{\partial F}\left[O_{1}(\tau(F))-O_{0}(\tau(F))\right]=-1 \\
\frac{\partial \tau}{\partial F}=-\frac{1}{\left[O_{1}(\tau(F))-O_{0}(\tau(F))\right]}>0
\end{gathered}
$$

which is always positive because, as previously stressed, $O_{1}<O_{0}$. This result demonstrates that $\tilde{\tau}$ is increasing in $\mathrm{F}$.

\section{Proof of Proposition 2:}

The Lagrangian of the minimization problem is given by:

$$
\begin{aligned}
L= & -n_{1}\left[\pi_{1}\left(O_{1}\right)-C_{1}\left(o_{1}\right)-F\right]-n_{0}\left[\pi_{0}\left(O_{0}\right)-C_{0}\left(o_{0}\right)\right] \\
& +D\left(n_{1} O_{1}+n_{0} O_{0}\right)-\lambda_{0} n_{0}-\lambda_{1} n_{1}-\mu\left(n_{0}+n_{1}-n\right)
\end{aligned}
$$

where $\lambda_{i}$ are the Kuhn_Tucker multipliers of the non negative constraints for $n_{1}$ and $n_{0}=n-n_{1}$. We denote $W=n_{1} O_{1}+n_{0} O_{0}$ as the total use of oil choosen by firms under taxation. Differentiating the A.5 respect to $O_{i}, n_{i}$ we obtain the first order conditions:

$$
\begin{gathered}
\frac{\partial L}{\partial O_{0}}=-\frac{\partial \pi_{0}}{\partial O_{0}}+\frac{\partial C_{0}}{\partial O_{0}}+\frac{\partial D(W)}{\partial O_{0}}=0 \\
\frac{\partial L}{\partial O_{1}}=-\frac{\partial \pi_{1}}{\partial O_{1}}+\frac{\partial C_{1}}{\partial O_{1}}+\frac{\partial D(W)}{\partial O_{1}}=0 \\
\frac{\partial L}{\partial n_{0}}=-\pi_{0}\left(O_{0}\right)+C_{0}\left(O_{0}\right)+\frac{\partial D(W)}{\partial W} O_{0}-\lambda_{0}-\mu=0 \\
\frac{\partial L}{\partial n_{1}}=-\pi_{1}\left(O_{1}\right)+C_{1}\left(O_{1}\right)+F+\frac{\partial D(W)}{\partial W} O_{1}-\lambda_{1}-\mu=0 \\
\text { eliminating } \mu \text { yields: } \\
-\pi_{1}\left(O_{1}\right)+\pi_{0}\left(O_{0}\right)+C_{1}\left(O_{1}\right)-C_{0}\left(O_{o}\right)+\left(O_{1}-o_{0}\right) \frac{\partial D(W)}{\partial W} \\
+F-\lambda_{1}+\lambda_{0}=0
\end{gathered}
$$

First, suppose that there exists an interior solution, i.e. $\lambda_{0}=\lambda_{1}=0$.

Differentiating system A.6 and A.7 w.r.t. $F$ and using the chain rule in A.7 we have the following equations:

$$
\begin{aligned}
& -\frac{\partial^{2} \pi_{0}\left(O_{0}\right)}{\partial O_{0}^{2}} \frac{\partial O_{0}}{\partial F}+\frac{\partial^{2} C_{0}}{\partial O_{0}^{2}} \frac{\partial O_{0}}{\partial F}+\frac{\partial^{2} D(W)}{\partial W^{2}} \frac{\partial W}{\partial F}=0 \\
& -\frac{\partial^{2} \pi_{1}\left(O_{1}\right)}{\partial O_{1}^{2}} \frac{\partial O_{1}}{\partial F}+\frac{\partial^{2} C_{1}}{\partial O_{1}^{2}} \frac{\partial O 1}{\partial F}+\frac{\partial^{2} D(W)}{\partial W^{2}} \frac{\partial W}{\partial F}=0
\end{aligned}
$$

$$
\frac{\partial^{2} D(W)}{\partial W^{2}}\left(O_{1}-O_{0}\right) \frac{\partial W}{\partial F}+1=0
$$

Given that $O_{0}>O_{1} \forall \tau$ we can write

$$
\frac{\partial^{2} D(W)}{\partial W^{2}}\left(O_{0}-O_{1}\right) \frac{\partial W}{\partial F}-1=0
$$

This yields

$$
\frac{\partial W}{\partial F}=\frac{1}{\frac{\partial^{2} D(W)}{\partial W^{2}}\left(O_{0}-O_{1}\right)}>0
$$

i.e. optimal aggregate use of oil is increasing in F. Substituting A.9 into A.8 yields:

$$
\frac{\partial O_{1}}{\partial F}=-\frac{1}{\left(O_{0}-O_{1}\right)} \frac{1}{\left[\frac{\partial^{2} C_{1}}{\partial O_{1}^{2}}-\frac{\partial \pi_{1}\left(O_{1}\right)}{\partial O_{1}}\right]}
$$

Unlike Requate and Unold [4] the Equation A.10 is 
negative only if: $\frac{\partial^{2} C_{1}}{\partial O_{1}^{2}}>\frac{\partial^{2} \pi_{1}\left(O_{1}\right)}{\partial O_{1}^{2}}$. and we assume this condition holds. So we have: $\frac{\partial O_{i}}{\partial F}<0 \quad \forall i=0,1$. Hence, optimal use of oil by each firm is decreasing in F.

Now total energy wasting can be written as

$$
W(F)=n_{1}(F) O_{1}(F)+n_{0}(F) O_{0}(F)
$$

Differentiating A.11 w.r.t. F and solving for $\frac{\partial n_{1}(F)}{\partial F}$ yields:

$$
\frac{\partial n_{1}}{\partial F}=\frac{+\frac{\partial W(F)}{\partial F}-\frac{\partial O_{1}(F)}{\partial F} n_{1}(F)-\frac{\partial O_{0}(F)}{\partial F} n_{0}(F)}{\left(O_{1}-O_{0}\right)}<0
$$

From Equation A.7 we know that

$$
\begin{aligned}
& C_{1}\left(O_{1}\right)-C_{0}\left(O_{o}\right)+\left(O_{1}-O_{0}\right) \frac{\partial D}{\partial W}-\left(\pi_{1}-\pi_{0}\right)+F= \\
& +\lambda_{1}-\lambda_{0}
\end{aligned}
$$

If $F$ is large, the LHS of the A.12 is positive since that $O \in\left[0, O_{\max }\right]$ so we have $\lambda_{0}=0$ and $\lambda_{1}>0$ $\rightarrow n_{1}=0$. So, when $F$ is large, there exists an $\bar{F}$ such that if $F \geq \bar{F} \quad$ no firm should adopt the advanced technology.

Let us rewrite Equation A.12 in the following way:

$$
\begin{aligned}
& {\left[\pi_{1}-C_{1}\left(O_{1}\right)-F\right]-\left[\pi_{0}-C_{0}\left(O_{0}\right)\right]-} \\
& \left(O_{1}-O_{0}\right) \frac{\partial D}{\partial W}=\lambda_{0}-\lambda_{1}
\end{aligned}
$$

if $F$ is very small we have $\left[\pi_{1}-C_{1}\left(O_{1}\right)-F\right]>$ $\left[\pi_{0}-C_{0}\left(O_{0}\right)\right]$ and the LHS of Equation A.13 is positive. This involves $\lambda_{0}>0$ that means $n_{0}=0$. So when $\mathrm{F} \approx 0$, there exists an $\underline{F}$ such that if $F \leq \underline{F}$ all firms adopt the advanced technology.

So far we have demonstrated that $n_{0}$ (and consequently $n_{1}$ ) is one for $F$ large and zero for $F$ small. However, being the functions of Equation A.13 continuos is $F$, there exist values for $F$ such that both $n_{1}$ and $n_{0}$ are outside the corner solutions: $\left[n_{1}=0, n_{0}=1\right]$ and $\left[n_{1}=1, n_{0}=0\right]$. So we conclude that for $F \in\lfloor\underline{F}, \bar{F}\rfloor$ we have $n_{1}, n_{0}>0$. In this case there is partial adoption.

\section{Proof of Proposition 3:}

When the regulator chooses the instrument (taxation) and fixes the instrument's level in the same time, there are three possible cases:

1) $F \leq F$

We have shown that in such a case $n_{1}=1$ and $n_{o}=0$ this means that F.O.C for the Equation A.5 involves :

$$
-\frac{\partial C\left(O_{1}\right)}{\partial O_{1}}+\frac{\partial \pi_{1}}{\partial O_{1}}=\frac{\partial D(W)}{\partial O_{1}}
$$

but for the firm F.O.C. we know that $-\frac{\partial C\left(O_{1}\right)}{\partial O_{1}}+\frac{\partial \pi_{1}}{\partial O_{1}}=\tau$. So finally, the government sets $\tau=\frac{\partial D(W)}{\partial O_{1}}$. By so doing, the optimal taxation is coherent with firm profit maximization and there is a subgame perfection.

2) $F \geq \bar{F}$

Similar analysis holds. In such a case $n_{1}=0$ and $n_{o}=1$ this means that F.O.C for the Equation A.5 involves :

$$
-\frac{\partial C\left(O_{0}\right)}{\partial O_{0}}+\frac{\partial \pi_{0}}{\partial O_{0}}=\frac{\partial D(W)}{\partial O_{0}}
$$

but for the firm F.O.C. we know that $-\frac{\partial C\left(O_{0-}\right)}{\partial O_{0}}+\frac{\partial \pi_{0}}{\partial O_{0}}=\tau$. So finally, the government sets $\tau=\frac{\partial D(W)}{\partial O_{0}}$. and the strategy is subgame perfect .

3) $\underline{F}<F<\bar{F}$

In this case the regulator sets $\tau$ by minimizing Equation A.5. This involves a particular value for $n_{1}$ and $n_{o}$. Nevertheless as firms play in the second stage in an uncoordinated way, there is no certainty that $n_{1}$ and $n_{o}$ will be effectively implemented. For the same reason, is possible that, for pure chance, firms choose $n_{1}$ and $n_{o}$ coherently with the optimal value chosen by the government. Hence multiple equilibria are possible, but only one is the efficient one. 